Service social

\title{
La dissociation et ses effets sur le " réseau de parenté »
}

\section{Didier LeGall et Claude Martin}

Volume 37, numéro 1-2, 1988

Par-delà les barrières des sexes

URI : https://id.erudit.org/iderudit/706394ar

DOI : https://doi.org/10.7202/706394ar

Aller au sommaire du numéro

\section{Éditeur(s)}

École de service social de l'Université Laval

\section{ISSN}

1708-1734 (numérique)

Découvrir la revue

\section{Citer ce document}

LeGall, D. \& Martin, C. (1988). La dissociation et ses effets sur le « réseau de parenté ». Service social, 37(1-2), 224-244. https://doi.org/10.7202/706394ar

\section{Résumé de l'article}

L'objet de cette recherche n'est pas d'évaluer l'impact de la séparation ou du divorce sur les acteurs de la famille, mais d'apprécier l'évolution du réseau parental à la suite d'un tel événement.

À l'aide d'une enquête postale, les auteurs cernent le profil des conditions de la dissociation et mettent en relief une typologie des configurations du réseau de parenté après la désunion. d'utilisation que vous pouvez consulter en ligne.

https://apropos.erudit.org/fr/usagers/politique-dutilisation/ 
LEGALL, Didier, chargé de recherche au

Centre de recherche sur le travail social, Université de Caen.

MARTIN, Claude, chargé de recherche au Centre de recherche sur le travail social, Université de Caen.

\section{La dissociation et ses effets sur le « réseau de parenté »}

\section{Didier LeGall Claude Martin}

Séparation, divorce, monoparentalité ou vie conjugale, chacune de ces situations renvoie à des expériences particulières, à des cas vécus ou connus et à un ensemble de valeurs et de pré-conceptions difficilement contournables. La fréquence des publications et débats (scientifiques ou médiatiques) ayant pour thème l'impact de la séparation ou du divorce sur les enfants, montre à quel point ce problème préoccupe nos sociétés contemporaines où ces situations se multiplient, voire se banalisent, tout en continuant de véhiculer nombre de stéréotypes et de préjugés.

Aujourd'hui, de plus en plus d'enfants sont susceptibles de connaître une période pendant laquelle ils seront séparés d'un de leurs parents. Voici les estimations de F. Furstenberg et de ses collaborateurs pour les États-Unis :

"À ne tenir compte que des enfants qui vivent chez un de leurs parents biologiques au moins, et non compris les enfants nés hors mariage, nous avons constaté que presque le tiers des enfants, au total, à quinze ans avait traversé une rupture familiale. Si on ajoute à cela les enfants nés hors mariage et les enfants qui ne vivent chez aucun de leurs parents, la proportion atteint environ $39 \%$, chiffre qui, selon nous, représente de façon plus exacte le véritable risque que les enfants ont de connaître un jour une famille monoparentale. Si l'on ajoutait à ce chiffre ceux qui traverseront la rupture après seize ans, la prévision serait que près de la moitié des enfants vivant aujourd'hui aux ÉtatsUnis n'auront pas le jour de leur dix-huit ans, vécu continûment entre leurs deux parents biologiques. " 1 
Pour la France, J.-L. Rallu estime que "les effectifs de familles (comprenant au moins un enfant) dont les parents ont divorcé passent

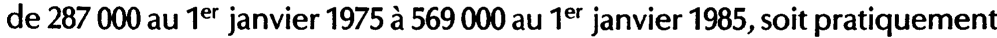
un doublement en 10 ans. [...] Ainsi, à 10 ans, près de $12 \%$ des enfants sont enfants de divorcés et près de $14 \%$ le sont à 16 ans. " 2 Ces chiffres ne tiennent pas compte des enfants dont les parents sont seulement séparés. À cela, il faut ajouter qu'en France, environ $14 \%$ des enfants naissent aujourd'hui hors mariage. On voit, par ces quelques estimations, que le phénomène est extrêmement important dans divers pays occidentaux et qu'il concerne un nombre croissant d'enfants.

Aborder l'impact de ces situations sur les enfants est très problématique. Peu de thèmes de réflexion sont autant imprégnés de charges émotionnelles et de valeurs morales, de risques de simplification et de difficultés méthodologiques, laissant libre champ à une multitude d'interprétations contradictoires. Peut-on, en ce domaine, effectuer des généralisations ? Quelles peuvent être les contributions des sciences humaines dans l'analyse de ce phénomène? Peuvent-elles se limiter à faire la preuve et l'inventaire des dégâts? Et, si oui, quels sont les effets sociaux de telles perspectives?

En effet, les approches traditionnelles portent sur les « risques » que ces "accidents de conjugalité » font encourir aux acteurs de la famille, mais aussi sur les manières d'intervenir pour faciliter leur adaptation. Selon les idéologies dominantes et selon les époques, la manière d'aborder ce sujet et les résultats des investigations sont radicalement différents. Si l'on en croit les plus récents commentaires, les mentalités auraient subi une véritable révolution. Le divorce d'aujourd'hui, par exemple, n'aurait plus grand chose à voir avec celui d'hier. Pourtant, les ambiguiités demeurent et les oppositions persistent entre progressisme et visions traditionnelles, entre propos alarmistes et tentatives de dédramatisation. Éviter de prendre parti dans un tel débat devient difficile. S'en tenir à une simple lecture des conditions de socialisation semble tout aussi délicat, tant est pressante la demande d'évaluation des effets (négatifs) et de résolution des problèmes associés à ces situations souvent complexes. N'est-ce pas d'ailleurs dans la mesure où elles peuvent provoquer des " problèmes sociaux » qu'elles font l'objet d'une attention aussi soutenue?

Certes, séparations et divorces sont des événements pénibles qui, du fait des difficultés relationnelles, entraînent souvent la recherche de divers supports extérieurs, que ce soit pour les adultes ou pour les enfants. Cet argument explique l'importance des travaux cliniques et évaluatifs qui, par ailleurs, nous semblent insuffisants pour rendre compte de la complexité de ces situations. À la limite, cette polarisation sur les dimensions cliniques occulte d'autres aspects de la réalité et 
renforce une certaine image à laquelle on peut accoler les termes de "problème social", de "handicap", de difficultés psychologiques, etc.

Contrairement à la tradition que nous venons d'évoquer, et qui privilégie les effets sur les personnes, nous avons récemment entrepris une recherche, dont le souci n'était pas d'évaluer l'impact de la séparation ou du divorce sur les acteurs de la famille, mais d'apprécier l'évolution du «réseau parental » à la suite de la séparation ou du divorce. ${ }^{3}$

\section{Des effets négatifs aux effets positifs selon les perspectives et les époques}

Entre les années cinquante et soixante-dix, de très nombreux travaux se sont penchés sur la question des effets de la dissociation familiale sur les membres de la famille, et notamment sur les enfants. Cette perspective est particulièrement développée dans la littérature anglo-saxonne. ${ }^{4} \mathrm{II}$ existe cependant un phénomène comparable en France, avec certaines particularités. ${ }^{5}$ Globalement, on peut dire que la grande majorité de ces travaux abordent la question en termes de problèmes d'ordre psycho-affectif ou cognitif.

Cette tendance peut s'expliquer de diverses manières. D'une part, on se doit de prendre en compte l'époque à laquelle nombre de ces démarches ont été entreprises, époque où la "famille nucléaire " était conçue comme le modèle unique, et toutes les variantes à ce modèle comme des déviances plus ou moins graves dans leurs effets. Cette première explication se réfère donc au rôle qu'ont pu jouer un certain nombre de valeurs dominantes concernant la famille, valeurs qui n'ont pas été sans influencer les orientations des chercheurs. D'autre part, ces travaux ont majoritairement adopté des démarches transversales, évaluatives et comparatives (comparaison avec des " familles normales " ou biparentales), portant sur la période qui suit plus ou moins immédiatement la séparation ou le divorce. Cette focalisation de l'attention sur l'épisode le plus critique, celui durant lequel les membres de la famille sont le plus affectés dans leurs personnalités et comportements, a eu, bien sûr, des effets considérables sur la manière d'envisager la séparation et le divorce. On n'a pas pu, semble-t-il, s'empêcher de poser certaines questions et d'en occulter d'autres, de privilégier certaines familles, d'omettre de prendre en compte certaines variables ou de se limiter à l'étude de certaines périodes particulièrement critiques. Il faudra attendre les années soixante-dix et quatre-vingt pour qu'apparaissent de nouvelles questions, et donc, de nouvelles réponses.

Il ne s'agit pas de dire pour autant, comme l'ont annoncé certains, que la famille nucléaire était en voie de disparition à cette époque. 
D'ailleurs, aujourd'hui « $80 \%$ des enfants de moins de 17 ans sont nés de parents mariés et vivent encore avec eux deux».6 Cependant, il est évident que le regard porté sur les contextes de socialisation que représentent les couples dissociés va dépendre de ces évolutions quantitatives, sur lesquelles prennent appui des valeurs et des normes. À la limite, plus ces situations sont marginales, plus elles risquent d'être analysées en termes déficitaires, voire stigmatisants. Un autre aspect entre en ligne de compte : les couches sociales de ces foyers. L'évaluation sera d'autant plus négative qu'elle concerne des milieux défavorisés où les causes des difficultés vécues par les adultes et les enfants sont multiples. À contrario, si, comme c'est le cas aujourd'hui, ces situations familiales se retrouvent dans toutes les couches sociales, les effets négatifs ont toute chance d'être moins manifestes, et donc les conclusions plus nuancées.

La lecture de ces situations familiales va connaître un important bouleversement au tournant des années soixante-quinze. À cette période riche en changements de toutes sortes (idéologiques, démographiques, juridiques, etc.) apparaît une nouvelle expression pour qualifier l'ensemble des situations où un seul parent élève son ou ses enfants: les "familles monoparentales". ${ }^{7}$ Cette expression, qui émerge dans les travaux de certains sociologues, exprime avant tout une nette volonté de déstigmatiser ces foyers, de leur donner une nouvelle image sociale d'alternative, de renouveau, et non plus de déviance. L'augmentation considérable du nombre des divorces à partir de cette date n'est pas sans jouer un rôle dans le renouvellement de l'image de ces situations familiales. Au fil du temps, cette expression va se diffuser, notamment dans le secteur des politiques sociales et du travail social, véhiculant une certaine image de misérabilisme. Les " familles monoparentales " seront peu à peu associées à une catégorie, à une population cible de l'intervention sociale, et à un état stable qui se conjugue avec précarité et pauvreté, négligeant la grande disparité des situations, en ne considérant que celles que rencontrent les intervenants sociaux. ${ }^{8}$

Autrement dit, si elle a permis une relative déstigmatisation de ces foyers, la catégorie " famille monoparentale " occulte un certain nombre d'aspects et, en particulier, leur mobilité. Les situations monoparentales ne sont pourtant bien souvent qu'une séquence de vie, un épisode de plus en plus "normal» des trajectoires familiales. À cet égard, il est important de noter la quasi-absence (en France) de travaux sur ce que l'on dénomme aujourd'hui la "recomposition" familiale. Alors que tout un ensemble de recherches se sont penchées, ces dernières années, sur les "familles monoparentales", tentant d'apprécier les difficultés qu'elles rencontraient, il semble que l'on ait totalement négligé la mobilité de ces situations qui, au fil du temps, se modifient, se 
recomposent et donc se complexifient, ce qui ne retire rien au fait qu'elles rencontrent un certain nombre de difficultés, notamment économiques. 9

En résumé, malgré l'évolution des perspectives, de nombreux aspects demeurent négligés; essentiellement : le fait que la séparation ou le divorce sont des événements inscrits dans une trajectoire que l'on s'interdit de comprendre lorsque l'on n'en étudie que la partie la plus visible. Comme l'écrit L. Roussel : «Étudier le divorce et ses conséquences, sans savoir de quel mariage le divorce est la rupture, c'est se condamner à n'observer, de l'histoire du couple, que la part la plus dramatique, mais peut-être la moins fondamentale. L'essentiel est sans doute en amont ". ${ }^{10}$ Mais il n'y a pas que l'amont de la séparation qui se trouve négligé. En effet, trop d'études se limitent à une coupe transversale au moment de la séparation et n'appréhendent pas les effets produits à long terme. En aval, beaucoup de choses se transforment et, tout particulièrement, le tissu des relations sociales de l'enfant ou encore les conditions matérielles de vie, les ressources et les modes d'existence quotidienne. Ainsi, contrairement à un nombre d'idées reçues, la rupture du lien conjugal n'entraîne pas nécessairement la rupture du lien parental. Les parents restent liés par l'existence de l'enfant, même si de nombreux bouleversements sont susceptibles d'intervenir et, notamment, le fait que l'un et/ou l'autre des parents vive(nt) une nouvelle relation conjugale. Autant de points qu'il nous semble nécessaire de privilégier.

\section{Des effets sur les personnes aux effets sur le réseau parental}

L'appréciation des effets du divorce ou de la séparation sur les enfants s'avère donc très complexe puisque, comme le note L. Rousseau, «il n'existe pas parmi les enfants une catégorie homogène dont le trait principal serait que leurs parents sont divorcés ". ${ }^{11}$ On sait aujourd'hui qu'un nombre important d'enfants sont et seront amenés à connaître un épisode de vie monoparentale. Ce type de constat révèle une mutation très importante des opinions et attitudes au sujet de ce que représente le lien biologique au sein de la famille, de même qu'en ce qui concerne les facteurs influant sur le maintien ou la rupture du lien conjugal et / ou parental. On ne peut désormais s'attacher à comprendre les effets et les raisons d'une dissociation sans tenir compte des raisons qui ont présidé à la constitution du couple. L'important à saisir n'est plus tant le moment de la dissociation que l'ensemble de la séquence qui la précède et qui la suit. ${ }^{12}$ 
Manifestement, c'est l'idée que nous nous faisons du couple, de la famille et de leurs fonctions qui a changé, modifiant ainsi les représentations que nous avons de la rupture et de la place de l'enfant dans la dissociation. Ainsi, la tendance particulièrement dominante dans les couches moyennes, à faire de la qualité de la relation conjugale le ciment de l'union, est déterminante dans l'approche de la place de l'enfant lors de la rupture de la famille. Certains adultes jugent positif le fait de se séparer, épargnant ainsi l'enfant de la faible qualité relationnelle du couple. Une telle attitude a un double impact. Le premier concerne la distinction entre rapport conjugal et rapport parental, l'un et l'autre pouvant très bien exister indépendamment. Le second renvoie à la conception que l'on se fait du lien biologique dans la socialisation de l'enfant, qui, à la limite, devient secondaire par rapport à la qualité du climat relationnel offert par les adultes, qu'ils soient ou non les géniteurs. Certains psychologues insistent d'ailleurs sur l'importance de la présence de deux partenaires auprès de l'enfant, qu'ils soient ou non les parents biologiques (en tous cas en ce qui concerne le père). Le remariage ou la cohabitation sont ainsi considérés, dans un certain nombre de recherches, ${ }^{13}$ comme ayant des conséquences bénéfiques pour l'enfant de familles dissociées.

L'évolution actuelle, suite au nouveau regard porté sur le couple, modifie assez radicalement la manière d'apprécier l'effet de la dissociation familiale sur l'enfant. II semble ainsi urgent d'abandonner le postulat déficitaire, qui vise essentiellement à mettre en évidence les difficultés ou handicaps immédiats provoqués sur l'enfant par la désunion des parents. En fait, beaucoup de variables entrent en ligne de compte, variables qui relèvent à la fois de la manière dont se déroulent tant l'amont que l'aval de la séparation, mais aussi de l'appartenance sociale. En revanche, les interactions et leurs modifications suite à la séparation, doivent être mieux appréciées.

$\mathrm{Si}$, certes, on enregistre un moins grand nombre de remariages, la reconstitution de couples après la séparation ou le divorce, sur un mode cohabitant ou non, se développe. ${ }^{14} \mathrm{~N}$ 'ayant que peu de données, en France, à ce sujet, nous ne pouvons préjuger de la quantité de ces cas, ni de la qualité relationnelle de ces unions et encore moins de leur durabilité. Cependant, cette tendance est susceptible de remettre en cause l'idée traditionnellement admise selon laquelle la dissociation induit une "réduction" du champ de sociabilité de l'enfant, dans la mesure où l'on passe d'une famille biparentale à une famille monoparentale. Par ailleurs, la manière dont les parents agencent leurs rapports afin de prendre en charge l'enfant suite à une désunion tend à se modifier, allant peut-être dans le sens d'un ajustement mutuel afin de ne pas faire de celui-ci la victime d'un choix plus ou moins négocié. 
Si l'enfant est au centre de ces réagencements de la structure familiale, il est urgent de mieux appréhender l'étendue de son champ de sociabilité, c'est-à-dire l'étendue des rapports qu'il se trouve amené à connaître suite à une séparation ou un divorce. Dans cette perspective d'analyse, il va de soi qu'au-delà du rapport enfant(s)-parent gardien, on ne peut évacuer la place que sont susceptibles de tenir un certain nombre d'acteurs significatifs : l'ex-conjoint, la famille du parent gardien, la famille de l'ex-conjoint (autrement dit, les grands-parents), l'éventuel nouveau partenaire et sa propre famille d'origine, sans parler des enfants de ces nouveaux acteurs, etc. Ainsi, loin de réduire le champ de sociabilité familiale, la dissociation est susceptible de l'élargir, en tous les cas de le complexifier: du point de vue de l'enfant, la "scène familiale» ne se limite plus alors au parent gardien et au parent non gardien.

\section{Présentation de la population enquêtée}

Afin d'apprécier l'évolution du réseau de parenté suite à la dissociation de la famille, nous avons réalisé une enquête postale auprès de parents-gardiens, séparés ou divorcés, ayant un ou plusieurs enfants, répertoriés par la Caisse d'allocations familiales (C.A.F.) du département du Calvados. Cela dit, vu notre orientation, il nous a semblé nécessaire d'exclure, d'une part, les bénéficiaires de l'Allocation de parent isolé (API) ${ }^{15}$ et, de l'autre, les allocations se trouvant dans cette situation depuis moins d'un an. Notre questionnaire portant, entre autres, sur les stratégies de cohabitation, il n'aurait pas été pertinent de retenir un sous-ensemble qui, par définition, du fait de la perception de l'API, ne pouvait nouer ou développer des rapports conjugaux. Afin de n'introduire aucun biais, nous avons préféré exclure ce sous-groupe. De la même manière, nous n'avons pas retenu les personnes n'ayant pas vécu au moins un an de séquence monoparentale : faute de recul, il leur est en effet souvent difficile de percevoir avec netteté la configuration du nouveau champ de sociabilité. De plus, sauf exception, il y a peu de chance que des stratégies conjugales aient pu s'amorcer, vu la proximité de la séparation.

Le questionnaire a été envoyé aux 4184 allocataires de la C.A.F. ayant ce profil. Les résultats présentés ici proviennent des réponses de 1085 personnes $(25.93 \%)$ dont nous avons vérifié la représentativité a posteriori. ${ }^{16}$ La population étudiée compte $80.85 \%$ de divorcé(e)s et $19.15 \%$ de séparé(e)s. ${ }^{17}$ II s'agit quasi exclusivement de femmes, les hommes représentant moins de $10 \%$ des répondants. Plus de six sur dix (67.04\%) ont une activité professionnelle. 
La majorité appartient à la catégorie socio-professionnelle ${ }^{18}$ "Employés» (59.69\%) et, dans une moindre proportion, à celle des "Artisans, commerçants et chefs d'entreprise» (29.61\%). Un peu plus d'une personne sur cinq fait partie de la catégorie des "Professions intermédiaires", et une très faible proportion (4.37\%) appartient aux "Cadres et professions intellectuelles supérieures". Notons encore qu'un peu plus de deux personnes sur dix ont un travail à temps partiel, les intérimaires ne représentant que $1.41 \%$. Dans l'ensemble, les niveaux de salaire ne sont donc guère élevés.

Presque tous les répondants ont entre 25 et 45 ans, la majorité ayant entre 35 et 39 ans. On notera néanmoins que les parents gardiens de moins de 29 ans représentent près de $14 \%$ de notre population, ce qui laisse entendre que bon nombre des séparés et divorcés appartenant aux jeunes générations ont eu une vie de couple relativement brève.

Dans l'ensemble, notre population a un capital scolaire peu élevé. Une personne sur cinq n'a aucun diplôme, un peu plus d'une sur quatre a un Certificat d'études primaires et / ou un Certificat d'aptitude professionnelle, et deux sur dix ont un Brevet élémentaire de premier cycle et/ou un Brevet d'étude professionnelle. Seulement $16.6 \%$ ont un niveau égal ou supérieur au Baccalauréat. ${ }^{19}$

\section{Conditions de la dissociation}

La très grande majorité (78.11\%) des séparé(e)s et divorcé(e)s se sont mariés durant la période de 1966-1980, sachant que pour près d'un tiers, il s'agit de la première moitié de la décennie 1970. Peu d'enquêtés avaient connu un second mariage (3.13\%). Dans l'ensemble, notre population s'est mariée relativement tôt : plus de huit sur dix se sont mariés avant 25 ans, et plus d'un quart avant 20 ans. L'âge au mariage peu élevé s'explique en partie par le fait qu'il s'agit essentiellement de femmes. La durée de vie en couple (temps écoulé entre le mariage et la séparation effective, et non le prononcé du jugement du divorce) complète et précise ce qui vient d'être énoncé : pour $36.25 \%$ des couples, la séparation a eu lieu entre cinq et neuf ans après le mariage, la valeur modale étant la huitième année. On notera néanmoins que, pour deux mariages sur dix, la vie en couple n'a pas atteint la cinquième année. Les séparations sont donc relativement précoces.

La rupture consommée, que se passe-t-il ? La plupart du temps, les ex-conjoints engagent les procédures visant à légitimer la rupture. Pour la grande majorité des divorcé(e)s (81.83\%), le jugement de divorce intervient moins de deux ans après la séparation, alors que pour $11.69 \%$ et 9.94\%, il n'est prononcé respectivement que 3-4 ans et 5-6 ans après. 
Un quart d'entre eux sont divorcés depuis moins de deux ans et, dans l'ensemble, notre population est divorcée depuis peu de temps (plus de six sur dix depuis moins de sept ans). Les procédures de divorce auxquelles ces personnes ont eu recours se répartissent comme suit : "divorce demandé par l'un et accepté par l'autre", 38.57\%; "consentement mutuel par requête conjointe", $24.85 \%$; "divorce par faute ", $22.63 \%$ et "divorce pour rupture de vie commune", $13.95 \%$.

Dans le cas des séparé(e)s, moins nombreux, la phase dite de "séparation " étant en cours, la rupture est plus proche dans le temps. Quatre sur dix sont séparés depuis moins de 2 ans, trois sur dix depuis 3 ou 4 ar s et un peu plus d'un sur dix depuis 5 ou 6 ans. On peut toutefois être étcnné de constater que plus de quatre sur dix (43.59\%) ne soient pas en instance de divorce. Quant à ceux qui sont actuellement en instance de divorce, un tiers ont opté our une procédure du type "divorce par faute ", près de trois sur dix pour un " divorce demandé par l'un et accepté par l'autre" et un peu plus de deux sur dix pour un "divorce pour rupture de vie commune». Le "consentement mutuel par requête conjointe" n'est, en revanche, guère prisé (15.45\%).

Les stratégies des séparé(e)s et des divorcé(e)s semblent donc très différentes du point de vue des procédures légales. Il se peut d'ailleurs fort bien que le fait d'être encore aujourd'hui dans la situation de séparé(e)s soit dû à certaines " complications » qui expliqueraient notamment que le divorce par faute soit la procédure la plus fréquente chez les séparé(e)s, alors qu'à l'inverse, chez les divorcé(e)s, la désunion n'ayant pas posé de problème majeur, la procédure dite du "consentement mutuel par requête conjointe » soit préférée à d'autres. Il faudrait, bien entendu, nuancer ces résultats en tenant compte de la disparité des pratiques de divorce selon les tribunaux et les juges.

Le choix de la procédure est aussi étroitement lié aux raisons de la séparation, problème particulièrement délicat à évaluer. Il existe rarement une cause isolée facilement repérable. C'est pourquoi nous avons parfois éprouvé quelques difficultés à décoder les réponses fournies à cette question ouverte. La raison la plus fréquemment évoquée, mais qui, avouons-le, n'est guère explicite, peut être synthétisée dans la formule: "vie commune impossible". À lire les réponses de certains parents gardiens, parfois assorties de commentaires, nous sommes en droit de penser que certaines désunions sont survenues de fait pour cette simple raison. En revanche, il semble bien que, pour d'autres, que ce ne soit pas le cas. La formule doit être lue à un second degré. Mais faute d'informations complémentaires, et pour éviter une interprétation " abusive ", nous nous en sommes tenus à ce qui était énoncé. D'où, sans aucun doute, une surreprésentation de cette cause (le tiers des cas). 
Une chose apparaît certaine cependant : l'adultère, l'alcoolisme et la violence (tant sur le parent gardien que, parfois, sur les enfants), constituent fréquemment les raisons à l'origine de la rupture. Notons d'ailleurs que ces trois facteurs, combinés ou non entre eux, sont souvent présents dans les réponses. Souvent invoquées (respectivement dans $25 \%, 18.5 \%$ et $15.7 \%$ des cas), ces causes expliquent sans doute en partie que la procédure de "divorce pour faute" soit encore si fréquemment retenue.

\section{Interactions au sein du réseau de parenté}

Suite à la désunion, le parent gardien, le plus souvent une femme, élève seul son ou ses enfants. Quels types de relations potentielles permet cette situation, la plus simple entre toutes? Pour l'enfant, sa famille se réduit-elle à ce foyer? Oui, s'il n'a pas connu son père. Et encore, c'est ne pas considérer que la famille puisse s'étendre à la parenté proche, par exemple, les grands-parents, qui constituent parfois, quand ils ne résident pas trop loin, un relais effectif : prise en charge des enfants et " caisse de solidarité " dès que les conditions économiques se dégradent. En revanche, si l'enfant a connu son père et entretient avec lui des relations selon les prescriptions strictes prévues en cas de divorce - le droit de visite - , force est de constater que les relations familiales vont au-delà de l'entité "foyer monoparental", et que le père, parent "non gardien", joue aussi un rôle dans le processus de socialisation de l'enfant. Comme le souligne fort pertinemment $N$. Lefaucheur, croire que, du point de vue des enfants, les relations familiales se limitent au foyer dans lequel ils résident, relève d'une certaine occultation due à la façon dont nos catégories ont été établies. ${ }^{20}$

Au-delà de la dissociation, des liens perdurent, et cela est vrai aussi en ce qui concerne les ex-conjoints. Les rapports de parentalité ne cessent pas toujours avec les rapports de conjugalité. Selon nos résultats, un tiers des parents gardiens n'ont plus aucune relation avec leur exconjoint; un sur deux maintient des relations, mais celles-ci sont réduites à ce qui concerne le(s) enfant(s); enfin, les autres (16.3\%) estiment que ces relations vont au-delà des rapports de parentalité puisqu'ils les qualifient de "plutôt bonnes dans l'ensemble, voir amicales". Près de $65 \%$ des parents ont donc maintenu des relations au-delà de la désunion. Pourtant, près de la moitié des parents non gardiens ont déjà recomposé une famille (dont $25 \%$ avec un partenaire ayant des enfants).

Parmi les parents gardiens dont les parents (ou l'un des deux) ne sont pas décédés, un peu plus de huit sur dix déclarent que leurs enfants 
voient très fréquemment ou assez souvent leurs grands-parents, un peu plus de $15 \%$ les voient plutôt rarement ou presque jamais, et $3 \%$ ne les voient jamais. En revanche, il semble que, du fait de la désunion, les enfants aient moins de relations avec les parents du parent "non gardien "; en effet, $19.4 \%$ des parents gardiens dont les beaux-parents (ou l'un des deux) ne sont pas décédés, déclarent que leur(s) enfant(s) les voient fréquemment ou assez souvent, $28 \%$ plutôt rarement ou presque jamais et plus d'un sur deux ne les voient jamais. Même si la dissociation affecte la relation " enfants-parents du parent non gardien ", on constate le maintien d'un réseau de relations nouées antérieurement. Ce type de configuration du champ de sociabilité familiale - que nous n'avons pas étendu aux collatéraux - ne vaut que pour le cas le plus simple : un parent gardien élevant seul son (ou ses) enfant(s).

Bien évidemment, la vie en situation monoparentale est, elle aussi, faite de rencontres. Dire cela, ce n'est pas autant ignorer qu'un certain nombre de parents gardiens sont confrontés à une forme de solitude proche de l'isolement socio-affectif. II semble d'ailleurs que le fait d'avoir su maintenir et / ou créer un réseau de relations au-delà de la rupture, et dans lequel on est "enchâssé", soit ici déterminant. Mais constater que les trajectoires sont moins stables, faites de ruptures et d'unions, et que sur le "marché matrimonial » un nombre croissant de personnes est prédisposé à essayer de (re)construire une histoire avec un autre partenaire, conduit à s'interroger sur les différentes formes de recomposition qui sont à l'œurre dans le cadre de la séquence dite monoparentale.

\section{Mise en relief des formes de recomposition}

D'emblée, nous sommes tentés de dire qu'il existe deux formes de recomposition, sachant qu'ainsi, nous occultons inéluctablement " celles qui ne se voient pas" et "celles qui ne se voient plus".

Les relations ponctuelles, voire éphémères, qui se nouent et se dénouent tout au long de la trajectoire d'un parent gardien, participent selon nous des essais de recomposition d'une certaine sociabilité conjugale, sans pour autant préjuger de l'intention des protagonistes. Nous rangeons ces relations dans les "formes de recomposition familiale qui ne se voient pas ". Les remariages, qui sont eux aussi l'aboutissement d'un processus semblable, font en revanche partie de "celle qui ne se voient plus ". Cela est très net en ce qui concerne la logique administrative. Une famille recomposée dans un cadre marital est une famille biparentale. Elle n'a donc pas d'existence en tant que famille spécifique, alors même que tout nous indique, du point de vue des relations qu'elle met en jeu, 
qu'elle n'est en rien réductible à une famille constituée à la suite d'une première union. Pour reprendre la terminologie de $\mathrm{N}$. Lafaucheur, ${ }^{21}$ nous pourrions dire qu'il n'y a pas véritablement de distinction entre "foyer biparental simple» (l'enfant vit avec ses deux parents non séparés) et "foyer biparental composé " (l'enfant vit avec le parent gardien et son nouveau conjoint qui peut lui-même avoir un ou plusieurs enfants dont il a ou non la garde, voire avec les enfants de cette nouvelle union). Or, il est bien évident que les relations familiales "débordent " le cadre du foyer ainsi recomposé.

Si l'on veut encore parler de «famille », il faut se départir de la vision "famille biparentale " et l'étendre à tous les acteurs qui constituent un des pôles autour duquel s'articule une relation. La « transformation de l'entité familiale » nécessite alors de recourir à une autre unité d'analyse qu'Irène Théry, s'appuyant sur la sociologie américaine, expose en ces termes :

"Le terme de "famille composée" (blended family) traduit ce déplacement fondamental, en désignant comme un ensemble la totalité du réseau familial dans lequel le foyer s'intègre comme composante [...] Ce n'est plus à partir des relations du Parent gardien et de leurs modifications [...] mais à partir des enfants et de leur circulation qu'est identifiée une famille composée. »22

C'est en partant de la dynamique des échanges que l'on peut avoir une idée des différentes configurations familiales et de leur évolution. L'avantage de cette perspective est d'inclure tous les agents qui concourent au processus de socialisation du ou des enfant(s) d'une famille ainsi recomposée. Le parent "non gardien », souvent délaissé dans certaines approches parce que constituant, selon les catégories classiques, un ménage d'une seule personne, retrouve alors une place à part entière dans ce réseau complexe de relations familiales. Cette place est d'ailleurs loin d'être négligeable, tout d'abord parce qu'il participe, dans la majorité des cas, à l'entretien de son ou ses enfant(s) ${ }^{23}$ et ensuite, parce que, du point de vue de l'enfant, le nouveau conjoint du parent gardien ne peut se substituer au parent absent. Comme le remarque Louis Roussel : "Sauf exception, les relations de familiarité quotidienne, avec le beau-père par exemple, sont d'un tout autre ordre que celles maintenues avec le père biologique et symbolique. Elles peuvent parfois les suppléer, jamais les remplacer ". ${ }^{24}$ Un foyer recomposé ne doit pas être assimilé à un foyer biparental simple. Mais ce type de famille n'est pas perçu comme le terme d'un processus de recomposition pour cette simple raison que les catégories administratives s'attachent plus à détecter le type de «structure familiale » que la « configuration du 
champ de sociabilité familiale", qui est pourtant le cadre de référence de l'enfant.

Entre " celles qui ne se voient pas " et " celles qui ne se voient plus", nous avons proposé deux autres formes distinctes et clairement repérables de recomposition : les relations qui se nouent, d'une part, sur le mode de la "conjugalité non cohabitante » et, d'autre part, sur le mode de la "conjugalité cohabitante".

Parce que "souterraine" dans ses manifestations, la conjugalité non cohabitante (chacun chez soi, bien qu'existent des relations affectives et sexuelles privilégiées qui perdurent) n'est presque jamais prise en compte, ou bien est assimilée aux formes de recomposition ponctuelles qui « ne se voient pas". Dans notre enquête, à la question «Avez-vous un partenaire régulier avec lequel vous ne cohabitez pas ? ", $26.5 \%$ des parents gardiens ont répondu par l'affirmative. II semble donc que ce soit à tort que l'on ait sous-estimé ce type de conjugalité. II reste néanmoins que l'on sait peu de choses sur cette forme particulière de "mise en couple». Est-ce le premier pas vers une cohabitation, est-ce une forme de ponctuel "prolongé " ou une forme "stable " qui satisfait le désir d'autonomie de chacun parce qu'elle permet d'avoir un " conjoint » sans pour autant se « remettre » stricto sensu en couple ? On remarquera que, proportionnellement, les parents gardiens ayant un capital scolaire élevé vivent plus souvent que les autres ce type de conjugalité.

Le phénomène de cohabitation est désormais passé dans les mœurs. II est cependant toujours délicat d'apprécier son ampleur puisqu'il s'agit d'une situation de fait, privée, qui ne donne lieu à aucun acte, excepté dans les cas où un certificat de concubinage est demandé. Les parents gardiens sont donc probablement peu enclins à déclarer ce qui, pour nous, constitue une des formes de recomposition familiale.

$8.4 \%$ des séparé(e)s et divorcé(e)s du Calvados ont déclaré vivre avec un nouveau partenaire, ${ }^{25}$ et si ces derniers sont principalement célibataires, $44 \%$ sont, eux aussi, séparé(e)s ou divorcé(e)s. Autrement dit, parmi les parents gardiens qui optent pour une forme de recomposition familiale sur le mode de la conjugalité cohabitante, un peu plus de quatre sur dix vivent avec un partenaire qui a lui aussi fait l'expérience de la vie maritale, et donc a connu un accident de conjugalité. Parmi ceux-là, un peu plus de quatre sur dix ont eu un ou plusieurs enfants dans le cadre de cette précédente union, mais la très grande majorité n'en a pas la garde. ${ }^{26} \mathrm{Au}$ total, en tenant compte de ces deux formes de recomposition, près de $35 \%$ des familles enquêtées sont reconstituées.

À la lumière de ces quelques données, on voit la nécessité, comme nous l'avons noté plus haut en ce qui concerne les remariages, de ne pas considérer ce type de foyer comme un foyer monoparental (perspective 
administrative en fonction de la logique de l'ayant droit) ou un foyer biparental reconstitué sur le mode cohabitant (perspective de la sociologie récente), mais comme un système complexe de relations, autrement dit une entité familiale "plurifocale». Et cela d'autant plus que les parents non gardiens se remettent apparemment plus souvent en couple que les parents gardiens.

\section{Configurations du réseau de parenté après la désunion}

La dissociation familiale renvoie souvent à un ensemble d'images quasi indestructibles; le fait que la désunion morcelle le champ des relations antérieures est au nombre de celles-ci. Les discours issus du domaine de l'action sociale ont largement contribué à façonner ce "prêt-à-porter» de la monoparentalité. À les écouter, le conjoint ayant la garde du ou des enfants est un "parent isolé».

Confrontés aux situations les plus extrêmes, les travailleurs sociaux, on le sait, ont souvent tendance, sans en avoir conscience, à étendre à l'ensemble des foyers monoparentaux, la vision qu'ils ont de ceux auprès desquels ils interviennent. ${ }^{27} \|$ est vrai que ces derniers sont plus " connus " parce qu'ils posent et vivent des problèmes cruciaux. Mais la prolifération des discours sur ces foyers en voie de précarisation, ainsi que l'écho qu'ils reçoivent, contribuent à rendre invisibles ceux qui ne relèvent pas de l'action sociale et constituent pourtant la grande majorité. Autre effet ambigu du traitement social : l'intervention catégorielle. Si un parent gardien connaît des problèmes, on puise dans la panoplie des mesures en faveur des "familles monoparentales" pour intervenir. $\mathrm{Ce}$ faisant, on tend à occulter ce qui a produit cette situation: ici, la désunion, qui met en scène deux protagonistes, les ex-conjoints.

Notre propos n'est pas de faire le procès de l'action sociale. II a simplement pour but de souligner l'ambiguïté d'une approche qui focalise son "regard" sur le seul parent gardien. Ambiguïté que l'on peut exprimer en ces termes: à ne voir que le parent gardien, on ne s'interroge pas sur les rapports que celui-ci entretient avec le réseau de parenté antérieur. Or, nous l'avons vu, bon nombre d'ex-conjoints maintiennent un rapport de parentalité. De la même manière, les enfants ont fréquemment des rapports avec les parents du parent gardien. Qu'en est-il alors du champ relationnel à la suite de la désunion ? Autrement dit quelles relations subsistent simultanément entre le parent gardien, et donc aussi ses enfants, et les membres du réseau de parenté antérieur? Telle est bien la question que l'on peut se poser dès lors que l'on ne considère pas la "séquence monoparentale" comme un état 
stable, mais comme un « moment » d'une trajectoire familiale qui évolue sans pour autant que les "anciens" protagonistes disparaissent. Nous nous proposons, pour terminer cet article, d'apporter quelques éléments de réponse à cette question.

La question définit l'orientation de la démarche: apprécier les différentes configurations que peut prendre le réseau de parenté après la désunion, en y intégrant tous les acteurs qui peuvent avoir un rôle et une place spécifique dans le processus de socialisation de l'enfant. Bien entendu, les ex-conjoints y tiennent la place la plus importante (rapports de parentalité). Mais aussi les grands-parents ; tout d'abord en raison de l'augmentation de l'espérance de vie à la naissance, ${ }^{28}$ ensuite parce qu'il semble bien que l'évolution récente des structures familiales ne se soit pas systématiquement traduite par une disparition des rapports entre générations. ${ }^{29}$

II serait néanmoins limité de s'en tenir à ces seuls acteurs. Certains parents gardiens reforment un couple, d'autres entretiennent un rapport privilégié avec un nouveau partenaire avec lequel ils ne cohabitent pas. Il convient donc ici de ne pas mettre à l'écart ces nouvelles relations car, du point de vue des enfants, elles jouent aussi parfois un rôle déterminant. Ces individus prendront donc place dans le champ relationnel en tant qu'acteurs d'un «nouveau réseau ».

Nous n'utiliserons cependant l'expression "nouveau réseau de parenté " - bien que cela puisse déjà paraître excessif — que dans les cas de cohabitation. II n'y a certes pas de reconnaissance officielle de la mise en couple, mais nous considérerons que le fait de vivre ensemble équivaut à un engagement, et donc institue et consacre l'union. Dans cette optique, la référence à la notion de "champ" ou "réseau de parenté " paraît raisonnable. $D$ 'ailleurs, le nouveau partenaire qui partage quotidiennement la vie du parent gardien et de ses enfants n'a-t-il pas la position de beau-père ou de belle-mère? En revanche, les relations nouées sur le mode de la conjugalité non cohabitante étant plus " informelles", voire même "souterraines" dans leurs manifestations, nous évacuerons tout vocable référant à la notion de "parenté ". II nous semble préférable en effet d'avoir recours à une dénomination plus " neutre ", c'est-à-dire qui ne préjuge en rien de la nature, de la forme et de l'avenir de cette relation. ${ }^{30}$ Nous nous proposons tout simplement d'employer l'expression " recomposition sur un mode non cohabitant ".

Précisons enfin que nous ne prenons pas en compte, ici, un certain nombre de relations, qui peuvent cependant être significatives tels les rapports avec les amis, le voisinage ou les collatéraux. ${ }^{31}$ Par ailleurs, nous nous situons résolument dans une optique quantitative qui a pour objectif principal de répondre à deux questions : 
- quelles sont les différentes formes de configurations du réseau de parenté à la suite de la désunion, sachant que nous prenons en compte, ici, la situation actuelle du parent gardien (vit seul, vit avec un nouveau partenaire ou recompose sur le mode de conjugalité non cohabitante)? ;

- quelles configurations du réseau de parenté trouvons-nous le plus fréquemment après la désunion?

Puisque nous avons abordé le problème en opérant une distinction entre "réseau de parenté antérieur", "nouveau réseau de parenté " et "recomposition sur un mode non cohabitant", nous présentons les résultats en ces termes, sachant qu'il nous faut aussi envisager les cas où ces relations n'existent pas ou alors sont réduites à leur plus simple expression: le parent gardien n'a de rapports qu'avec ses propres parents. ${ }^{32}$ Un tel angle d'attaque nous amène à présenter grosso modo sept formes particulières du réseau de parenté. ${ }^{33}$

1. Le parent gardien vit seul, n'a aucune relation avec le réseau de parenté antérieur ${ }^{34}$ et n'entretient aucun lien de conjugalité avec un nouveau partenaire : 66 répondants, soit $6.09 \%$.

2. Le parent gardien, qui vit seul et ne recompose pas sur le mode non cohabitant, n'a des relations qu'avec ses parents : $11.91 \%$, soit 129 répondants.

3. Le parent gardien, qui vit seul et ne recompose pas sur le mode non cohabitant, a des relations avec le réseau de parenté antérieur : $50.88 \%$, soit 551 répondants.

4. Le parent gardien, qui recompose sur le mode non cohabitant, a des relations avec le réseau de parenté antérieur : $18.84 \%$, soit 204 répondants.

5. Le parent gardien vit avec un nouveau partenaire, a des relations avec le réseau de parenté antérieur et avec un nouveau réseau, qui inclut le nouveau partenaire et, parfois, ses parents : $6.56 \%$, soit 71 répondants.

6. Le parent gardien vit avec un nouveau partenaire et n'a des relations qu'avec le nouveau réseau de parenté (nouveau partenaire et parfois, les parents de celui-ci) : $1.57 \%$, soit 17 répondants. Ils ne maintiennent donc plus aucune relation avec le réseau de parenté antérieur.

7. Le parent gardien recompose sur le mode de la conjugalité non cohabitante, mais n'a plus aucune relation avec le réseau de parenté antérieur : $4.15 \%$, soit 45 répondants. 
À la lumière de ces résultats, quelques tendances globales se dessinent.

Les parents gardiens que nous pourrions qualifier d' " isolés", en ce sens qu'ils n'ont aucune relation avec au moins un des acteurs du réseau de parenté, tel que nous l'avons défini, sont peu nombreux $(6.09 \%)$. Pour eux cependant, la rupture semble consommée. Un peu plus d'un sur dix des répondants $(11.91 \%)$ sont dans la même situation, mais ont des relations (quelles qu'en soient la fréquence et la qualité) avec leurs parents. Ces deux configurations particulières du champ relationnel après la désunion semblent illustrer parfaitement le foyer monoparental, au sens où l'entend l'action sociale.

D'autres encore, bien que peu nombreux (5.72\%), n'ont plus de relations avec le réseau de parenté antérieur. Leur situation est cependant sensiblement différente. En effet, si la rupture, là aussi, semble consommée, ils ont noué de nouvelles relations : $4.15 \%$ avec un nouveau partenaire sur le mode de la conjugalité non cohabitante et, une infime minorité $(1.57 \%)$, avec un «nouveau réseau de parenté ».

En cumulant les formes 1, 2, 6 et 7 de la liste qui précède, un peu plus de deux parents gardiens sur dix $(23.72 \%)$ ne maintiennent plus aucune relation avec le réseau antérieur. II semble que la désunion des parents produise, pour un nombre de cas restreint, un morcellement du champ relationnel. Ce constat ne vaut cependant que pour le parent gardien. Il se peut en effet que les enfants aient encore des rapports avec le parent non gardien et, éventuellement, ses parents. Mais il est clair que ces relations s'entretiennent sans la médiation du parent gardien, ce qui ne constituerait pas le meilleur contexte de socialisation de l'enfant. Dans de tels cas, le "mode de circulation » des enfants a été étudié et institué de façon à ce que les ex-conjoints ne se rencontrent pas. Situation qui dénote un climat potentiellement conflictuel dès lors que le parent non gardien n'habite pas trop loin, n'est pas incarcéré ou décédé.

Dans tous les autres cas $(76.28 \%)$, des liens sont maintenus avec le réseau de parenté antérieur. Le plus souvent, ce rapport " transite" par le parent non gardien, ce qui favorise le maintien de relations avec les parents de celui-ci quand ils ne sont pas décédés. Mais dans certains cas, beaucoup plus rares, les rapports avec le réseau antérieur n'impliquent pas le parent non gardien. Ils se limitent en effet aux rapports "parent gardien - parents du parent non gardien ".

Affinons encore ce point en notant que, parmi ceux qui maintiennent un rapport avec le "réseau antérieur», près de deux sur dix $(\mathbf{1 8 . 2 4 \% )}$ recomposent sur le mode non cohabitant, et $6.56 \%$ vivent avec un nouveau partenaire. Cette dernière configuration, assez rare, illustre parfaitement la notion de « carrière familiale » qui renvoie à une trajectoire 
faite d'unions et de désunions, sans pour autant que les acteurs d'une union précédente disparaissent systématiquement du champ des relations dès qu'il y a remise en couple. On assiste alors à une sorte de " couplage " du réseau de parenté antérieur avec le nouveau, le parent gardien et ses enfants constituant le lien entre ces deux réseaux. Dans ce cas, loin de restreindre le champ de sociabilité de l'enfant au seul parent gardien et, éventuellement, aux parents de celui-ci, comme on le pense parfois, la désunion l'étend à de nouveaux acteurs.

Enfin, ces résultats nous renseignent sur deux points qui constituent, d'une certaine manière, la réponse aux questions que nous nous posions.

1. Selon la perspective privilégiée dans notre étude, il existe sept configurations différentes du champ de parenté à la suite de la désunion, dès lors que l'on intègre la recomposition sur le mode de la conjugalité non cohabitante. Ces sept profils vont de l'absence totale de relations (isolement) jusqu'au couplage d'un réseau antérieur avec un nouveau (extension du champ relationnel), donc du foyer monoparental comme figure de l'action sociale au foyer recomposé (toujours répertorié néanmoins comme " monoparental » par les institutions sociales) qui n'a pas rompu ses liens avec les protagonistes du réseau antérieur, en passant par diverses formes qui élargissent plus ou moins le champ de sociabilité de l'enfant.

2. La configuration du réseau de parenté que nous retrouvons le plus fréquemment $(50.88 \%)$ après la désunion est celle constituée par un parent gardien qui vit avec ses enfants sans avoir noué de nouvelles relations conjugales, mais maintient des rapports avec l'ancien réseau de parenté, sachant bien, toutefois, que l'on ne peut apprécier ici la qualité de ces relations.

Bien sûr, ces résultats nécessiteraient de poursuivre les investigations qualitatives, afin de mieux appréhender la nature de ces différentes relations et de les identifier en fonction des classes sociales. C'est dans cette perspective que nous nous situons désormais.

\section{Notes}

1 F.F. Furstenberg, C. Winquist Nord, J.L. Peterson et N. Zill; " L'itinéraire des enfants du divorce : rupture conjugale et contacts des enfants avec le parent non gardien ", Dialogue, "Les beaux-enfants. Remariages et recompositions

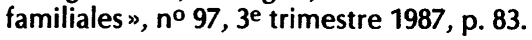


2 J.-L. RAllu : "Quelques estimations statistiques", Du divorce et des enfants, INED, Ministère de la Justice, PUF. 1985, pp. 15 et 19. ("Cahiers n० 111 ».)

${ }^{3}$ Cette recherche actuellement en cours a été financée par la Caisse nationale des allocations familiales. Elle a pour l'instant donné lieu au rapport suivant : D. LEGALl et C. MARTIN : Le champ de sociabilité familiale à la suite d'une désunion, Enquête postale auprès des séparé(e)s et divorcé(e)s de la C.A.F. du Calvados, Rapport intermédiaire, septembre 1987. (Le rapport final est disponible depuis avril 1988.)

4 Voir l'excellent bilan de littérature effectué par G. NeLSON : "Family adaptation following marital separation / divorce : a literature review ", dans : B. SCHLESINGER, The One-Parent Family in the 1980s. Perspectives and Annotated Bibliography 1978-1984, Toronto, University of Toronto Press, 1984 : 97-151.

5 En France, ce sont plutôt les pédopsychiatres qui vont développer cette perspective dans le cadre de l'étiologie de la délinquance. G. HEUYER n'écrivaitil pas, dans son Introduction à la psychiatrie infantile (Paris, PUF, 1966. ("Sup. »)): "Ce n'est pas sans des raisons profondes que toutes les statistiques établissent que $80 \%$ à $90 \%$ des délinquants juvéniles proviennent de foyers dissociés. Ce chiffre, par son ampleur même, montre les profondes conséquences de la rupture du cadre normal de la famille dans l'adaptation sociale de l'enfant $"$.

$6 \mathrm{H}$. Léridon et C. Villeneuve-Gokalp : "Entre père et mère", Population et sociétés, n०220, janvier 1988.

7 Voir: D. LeGall et C. Martin, Les familles monoparentales. Évolution et traitement social, Paris, ESF, 1987.

8 Voir: N. Lefaucheur: "Familles monoparentales: les mots pour les dire", dans: F. Bailleau, N. Lefaucheur et V. Peyre, Lectures sociologiques du travail social, Paris, CRIV / Éditions Ouvrières, $1985:$ : 215-215. ("Politiques sociales ».)

9 Voir : B. BASTARD et L. CARDIA-VONËCHE : "La situation économique des familles à un seul parent. Réflexion sur l'appauvrissement ", Les Cahiers médicosociaux, "Les familles monoparentales", $n^{\circ} 2,1987$.

10 L. Roussel : "Préface », dans : O. Bourguignon, J.L. Rallu et I. ThérY, Du divorce et des enfants, op. cit., p. 10.

11 L. ROussel, ibid.

12 Voir à ce sujet l'article de R. LefrançoIs : "Cadre familial et délinquance", Service social, "Déviance, délinquance et réaction sociale", vol. 33, nos 2-3, 1984 : 171-185.

13 Voir : T.S. PARISH et B.M. KAPPES, "Impact of father loss on the family ", Social behaviour and personality, n० 8, 1980 ; et T.S. PARISH et J.W. DOSTAL, "Evaluation of self and parent figures by children intact, divorced and reconstituted families ", Journal of youth and adolescence, n ${ }^{\circ}$ 9, 1980.

14 Voir: I. THÉRY : "Remariage et familles composées", L'année sociologique, "Sociologie de la famille 1965-1985", vol. 37, 1987. Voir aussi le numéro de la revue Dialogue : "Les beaux-enfants. Remariages et recompositions familiales", no 97, 3e trimestre 1987.

15 L'Allocation de parent isolé (API) a été créée en 1976 pour garantir temporairement un minimum de revenu mensuel aux parents seuls pour élever leurs enfants. Elle est versée dans la quasi-totalité des cas à des femmes, soit enceintes (elles en bénéficient alors jusqu'au trois ans de l'enfant), soit 
veuves, divorcées, séparées de fait ou de droit ou concubines abandonnées (dans ce cas l'allocation est versée pendant douze mois ou tant que le dernier enfant n'a pas atteint trois ans). En tant qu'application du principe du revenu minimum garanti, l'API complète les ressources (salaires, pensions, etc.) jusqu'à concurrence d'un plafond défini en fonction du nombre d'enfants à charge. Elle cesse d'être versée dans le cas où la bénéficiaire vit avec un partenaire.

16 Précisons dès à présent que cette population d'enquête n'inclut pas tous les séparés et divorcés du Calvados: tout d'abord, parce que nos critères en excluent un certain nombre et, ensuite, parce que ne sont pas pris en compte ceux qui relèvent de régimes spéciaux de sécurité sociale, tels les régime agricole, celui des transports, des Mines, le Commissariat à l'énergie atomique, la Banque de France, les marins du commerce et de la navigation intérieure, la pêche maritime et l'État. Du fait de ces régimes spéciaux, les résultats de notre enquête ne peuvent être généralisés à l'ensemble de la population. Ils ne valent que pour les séparés et divorcés de la CAF du Calvados, qui regroupe essentiellement les allocataires de l'industrie et du commerce, de la santé et de petites et moyennes entreprises. Inéluctablement, ces limites ont des répercussions sur les résultats de l'enquête. Ainsi, par exemple, le seul fait de ne pas prendre en compte les séparés et divorcés travaillant dans le secteur de l'Éducation nationale a pour effet direct de " baisser » le capital scolaire moyen de notre population.

17 La sous-représentation apparente des séparé(e)s tient à la définition de la catégorie elle-même : catégorie englobante en ce qui concerne la CAF qui regroupe ici l'ensemble des personnes devenues seules, qu'elles soient mariées ou non, alors que, pour les besoins de la recherche, nous l'avons limitée aux seuls séparé(e)s issu(e)s d'un couple légitime.

18 Il s'agit des catégories socio-professionnelles de l'Institut national de la statistique et des études économiques, Nomenclature 1982.

19 Rappelons, pour mémoire, qu'il s'agit ici du diplôme de fin d'études secondaires et non du diplôme de premier cycle universitaire québécois.

20 Voir: N. Lefaucheur, "Les familles monoparentales n'existent pas, je les ai rencontrées..." "; les Cahiers médico-sociaux, 1987.

21 Voir: N. Lefaucheur, "Les conditions et niveaux de vie des enfants de parents séparés ", Les enfants de parents séparés, Paris, IDEF-CTNERHI, 1987.

22 L. THÉRY, "Remariages et familles composées », L'année sociologique, op. cit., pp. 143-144.

23 Dans notre enquête postale sur les séparé(e)s et divorcé(e)s, près de six parents gardiens sur dix déclarent percevoir une pension alimentaire.

24 L. Roussel, "Préface de l'ouvrage ", Du divorce et des enfants, op. cit., p. 7.

25 Cette proportion peut paraître peu élevée. Elle s'explique en partie par le fait que la majorité des séparé(e)s et des divorcé(e)s vivent seul(e)s depuis moins de quatre ans, mais aussi sans doute pour la raison que nous venons d'évoquer. Cohabiter, c'est justement vivre avec quelqu'un sans pour autant faire reconnaître l'union ainsi constituée par la société. Elle appartient donc au registre du privé. II n'y a alors pas lieu non plus d'en faire état dans un questionnaire qui, bien que garantissant l'anonymat, a été diffusé avec la collaboration d'une CAF. 
26 Ce qui n'a rien d'étonnant ici puisque la garde des enfants est presque toujours attribuée à la femme et que, dans le cas présent, les nouveaux partenaires sont presque toujours des hommes.

27 Voir: D. LeGall et C. MARtin, Mouvance de la famille : réponses de l'action sociale, Caen, Centre de recherche sur le travail social, 1983.

28 Avec une espérance de vie, à la naissance, de 71,2 ans pour les hommes et 79,3 ans pour les femmes, en 1984, la France se situe aux tous premiers rangs des pays développés. L'augmentation progressive de l'espérance de vie accroît inéluctablement la probabilité que grands-parents et petits-enfants se connaissent et donc tissent des rapports, bien que la coupure entre générations se soit accentuée : "en 1962, les personnes âgées vivant seules étaient moins nombreuses que celles vivant avec des proches; en 1982, elles sont deux fois plus nombreuses" (P.-A. AUDIRAC, "Les personnes âgées et leur famille", Données sociales, Paris, INSEE, 1987, p. 530.)

29 A. Pitrou, "Dépérissement des solidarités familiales ? ", L'année sociologique, op. cit., pp. 207-224.

30 Cette précaution peut paraître elle aussi excessive. Cependant, travaillant par ailleurs avec des étudiants sur les formes de recomposition à la suite d'une désunion dans le milieu enseignant, nous avons eu plusieurs fois affaire à des relations sur ce mode qui nous interdisaient même d'avoir recours à la notion de "recomposition». Le cas type nous est fourni par une femme, divorcée depuis quinze ans, qui maintient depuis près de dix ans une relation privilégiée avec un père de famille. On peut ne voir ici qu'une situation vaudevillesque. Pourtant, cette relation joue un rôle déterminant dans la vie de cette femme, qui en a informé ses enfants. Ayant elle-même vécu douloureusement la désunion, elle refuse que son partenaire envisage toute séparation. Il est donc clair qu'il n'est pas question dans ce cas de "recomposition". Pourtant, l'expression "conjugalité non cohabitante» recouvre ou englobe ce type de relation qui ne réfère, selon nous, ni à une situation vaudevillesque, ni stricto sensu à une forme de recomposition familiale. Cas marginal si l'on veut, que nous avons aussi retrouvé chez une autre divorcée appartenant à la même tranche d'âge : 45-55 ans.

31 Voir, à ce sujet, le récent travail de A. Fortin: Histoires de familles et de réseaux. La sociabilité au Québec d'hier à demain, Montréal, Saint-Martin, 1987.

32 Par réseau de parenté antérieur nous entendons principalement les acteurs de la famille par alliance, à savoir l'ex-conjoint et ses parents. Nous excluons volontairement de ce réseau les parents du parent gardien dans la mesure où ces rapports ne sont pas noués lors de la phase conjugale, mais bien avant.

${ }^{33}$ Ces résultats portent sur 1083 réponses, deux enquêtés n'ayant répondu à aucune des treize questions sélectionnées pour apprécier ces différentes configurations.

${ }^{34}$ Ce qui ne signifie pas que les enfants ne voient pas, même ponctuellement, leur parent non gardien. 